\title{
SCHWERPUNKT: DIE PHILOSOPHIE VON RUTH G. MILLIKAN
}

Ruth Millikans Philosophie ist in den Traditionen der analytischen Philosophie und des klassischen amerikanischen Pragmatismus verwurzelt. Sie setzt sich kritisch mit Frege, Russell oder Strawson auseinander, übernimmt Einsichten von Wittgenstein, Quine oder Putnam, und sie steht spürbar unter dem Einfluss von Sellars. ${ }^{1}$ Seit dem Erscheinen ihres ersten Buchs Language, Thought, and Other Biological Categories hat Millikan in kontinuierlicher und kreativer Arbeit substanzielle Beiträge zu einer ganzen Bandbreite von Feldern der theoretischen Philosophie geleistet. ${ }^{2}$ Diese Beiträge betreffen nicht nur die Philosophie des Geistes oder die Philosophie der Biologie, sondern ebenso die Sprachphilosophie, Ontologie und Erkenntnistheorie.

Millikans wohl bekanntester Beitrag ist eine anspruchsvolle Version der sog. „Teleosemantik“, die von ihr selbst als „Biosemantik“ bezeichnet wird. ${ }^{3}$ Was ist Teleosemantik? Die Teleosemantik versucht eine Antwort auf eine altehrwürdige philosophische Frage zu geben, der Kant folgende Form gegeben hat: „[A]uf welchem Grunde beruhet die Beziehung desienigen, was man in uns Vorstellung nennt, auf den Gegenstand?“4 Der Ausdruck „Vorstellung“ ist eine Eindeutschung von „repraesentatio“"; „Verstand“ ist „,intellectus“, also das Vermögen, Vorstellungen hervorzubringen. In der gegenwärtigen Philosophie des Geistes spricht man weniger von „Vorstellungen“" und „Verstand“ als von „Repräsentation“, „Geist" und „mentalen Repräsentationen“. Man darf deshalb Kants Frage reformulieren: Auf welchem Grund beruht die Beziehung einer mentalen Repräsentation auf ihren Gegenstand? Anders gefragt: Worin besteht das Wesen der Intentionalität?

Die Teleosemantik findet den „Grund“ der Intentionalität im Begriff der Funktion. ${ }^{6}$ Es ist die Funktion - teleologischer gesagt: der Zweck - von Repräsentationen, sich auf diesen oder

1 Zu Sellars vgl. R. Millikan, The Son and the Daughter: On Sellars, Brandom, and Millikan, in: dies., Language. A Biological Model, Oxford 2005, Kap. 4. Zur Verankerung in der Tradition des Pragmatismus vgl. den Beitrag von Wolfgang Detel in diesem Heft. Zu Millikans Weiterführung der Projekte von Peirce und Sellars vgl. M. Wild, Biosemantik. Repräsentation, Intentionalität, Norm, Habilitationsschrift, HU Berlin, 2010. Für Beziehungen zwischen der Biosemantik und Peirce vgl. Th. L. Short, Peirce's Theory of Signs, Cambridge 2007.

2 Dazu gehören insbesondere Millikans fünf Bücher: dies., Language, Thought, and Other Biological Categories, Cambridge 1984; dies., White Queen Psychology and Other Essays for Alice, Cambridge 1993; dies., On Clear and Confused Ideas. An Essay about Substance Concepts, Cambridge 2000; dies., Varieties of Meaning, Cambridge 2004; dies., Language. A Biological Model, Oxford 2005. Eine sehr gute Einführung ist: N. Shea, On Millikan, Belmont 2005.

3 Vgl. R. Millikan, Biosemantics, in: The Journal of Philosophy, 86 (1989), 281-297 (wieder abgedruckt in: dies., White Queen Psychology, a. a. O., Kap. 4); dies., Biosemantics, in: Oxford Handbook of the Philosophy of Mind, hg. v. B. McLaughlin, Oxford 2009, 394-406.

4 Brief an Marcus Herz vom 21.2. 1772; AA X, 130.

$5 \mathrm{KrV}, \mathrm{A} 320 / \mathrm{B} 367$.

6 Millikan spricht von ,proper function“ (Eigenfunktion), vgl. dazu den Beitrag von Matthias Vogel in diesem Heft. Natürlich wirft diese Behauptung die Frage auf, ob Kant und Millikan hier im selben 
jenen Gegenstand zu beziehen. Ebenso wie Dinge, die ihre Funktionen bisweilen ausüben, bisweilen aber auch nicht funktionieren (weil sie defekt sind oder weil die Umstände die Funktionsausübung nicht zulassen), können auch Repräsentationen ihre Funktion bisweilen ausüben und bisweilen nicht. Es ist dieser Aspekt, der dafür sorgt, dass Repräsentationen korrekt oder unkorrekt, wahr oder falsch sein können. Die Funktion stellt also ein Maß - eine Norm im ursprünglichen Wortsinn - zur Verfügung, von dem eine Repräsentation abweichen kann, und verspricht so, die normative Dimension intentionaler Gehalte zu fassen. Vermöchte man nun den Begriff der Funktion auch noch auf einen natürlichen, nämlich biologischen, Grund zurückzuführen, so hätte man ein vielversprechendes Rezept für die Naturalisierung der Intentionalität. Genau dies will die Teleosemantik. Sie versteht repräsentationale Funktionen als biologische Funktionen (oder in Analogie ${ }^{7}$ zu biologischen Funktionen) - etwa wenn wir sagen, es sei die Funktion des Herzens, Blut zu pumpen. Die Teleosemantik kann so als naturalistische Antwort auf Kants Frage verstanden werden.

Die Teleosemantik wird in der Regel als Theorie des mentalen Gehalts charakterisiert. ${ }^{8}$ Dies ist im Hinblick auf Millikans Philosophie zwar nicht verkehrt, hat aber zu Verengungen geführt, die der Breite dieser Philosophie nicht gerecht werden. Es ist ein Ziel dieses Schwerpunktes, Millikans Philosophie möglichst in ihrer Breite vorzustellen, um sie einer Diskussion zugänglich zu machen. Entsprechend behandeln die Beiträge ein weites Themenspektrum. Doch von welchen Verengungen ist die Rede? Vier sollen genannt werden, bevor ein kurzer Blick auf die Beiträge diese Einführung abschließen wird. Erstens versteht sich die Biosemantik nicht nur als Theorie der Gehalte vorbegrifflicher oder begrifflicher mentaler Repräsentationen, sondern als Theorie von intentionalen Inhalten überhaupt, insbesondere auch der sprachlichen Bedeutung - schließlich bezeichnet der Titel von Millikans Erstling sowohl Sprache als auch Denken als biologische Kategorien. Dabei ist die Intentionalität der Sprache nicht konstitutiv von der Intentionalität des Denkens abhängig (oder vice versa), sondern Sprache und Denken verhalten sich - wie Millikan sagt - „weitgehend parallel zueinander".? Zweitens könnte Millikans in einem weiten Sinn biologische Auffassung der Intentionalität zu der irrigen biologistischen Auslegung verleiten, sie führe die Inhalte von Überzeugungen oder Aussagen entweder direkt auf unmittelbare biologische Bedürfnisse von Denkern oder Sprechern zurück, oder sie behaupte, dass begriffliche Repräsentationen angeboren seien. Weder das eine noch das andere trifft zu. ${ }^{10}$ Das Thema des Nativismus führt zum dritten Punkt. Bisweilen wird Millikans Biosemantik in die Nähe von Fodors Psychosemantik gerückt. Und so werden Einwände gegen die Psychosemantik gerne auf die Biosemantik übertragen. ${ }^{11}$ Nun verfolgen zwar beide das Ziel einer Naturalisierung der Intentionalität, doch die Unterschiede sind so gravierend, dass eine Parallelisierung irreführend wird. Einige dieser Unterschiede seien summarisch aufgeführt: Während Millikan jegliche Annahme eines unmittelbar Gegebenen entschieden zurückweist, tritt Fodor als Verteidiger des Gegebenen auf. Während Fodor

Sinn nach dem „Grund“ der Intentionalität von Repräsentationen suchen.

7 Vgl. dazu das Interview mit Ruth Millikan in dieser Nummer, 981-1000.

8 „There are a number of different teleological theories of mental content [...] it's central to all of them that a certain normative notion of function underwrites a certain normative notion of content." (K. Neander, Biological Approaches to Mental Representation, in: Handbook of the Philosophy of Science: The Philosophy of Biology, hg. v. M. Matthen u. C. Stevens, Amsterdam 2006, 550)

9 Vgl. R. Millikan, Language, a. a. O., 92.

10 Vgl. Millikans Replik auf Elder und das Interview mit Ruth Millikan in dieser Nummer, 975-979; 981-1000.

11 So etwa H. Putnam, Renewing Philosophy, Cambridge/Mass. 1992, Kap. 2-3. 
den semantischen Internalismus und den engen Gehalt verteidigt(e), ist Millikan eine extreme semantische Externalistin. Während Millikan intentionale Zustände als etwas betrachtet, das Gegenstand einer nicht-nomologischen Biosemantik ist, sieht Fodor intentionale Zustände als Objekte einer nomologisch verfassten kognitiven Psychologie. Während Millikan auf Darwins Theorie der natürlichen Selektion zurückgreift, um Kants Frage zu beantworten, lehnt Fodor jeglichen Rückgriff dieser Art ab. Die These der Biosemantik lautet, dass die Intentionalität subpersonaler, tierlicher, mentaler oder sprachlicher Repräsentationen biologischen Systemen in einem weiten Sinne zukommt, nämlich Systemen mit echten Funktionen. Aber Millikan - und das ist der vierte Punkt - bindet diese These stets eng an ontologische Überlegungen. So befassen sich die ersten drei Teile von Language, Thought, and Other Biological Categories mit der Theorie der Funktion, der intentionalen Zeichen und der sprachlichen Bedeutung. Der vierte Teil aber befasst sich mit Fragen der Identität von Substanzen, Eigenschaften und Individuen. In On Clear and Confused Ideas legen die ersten sieben Kapitel Millikans Theorie der Substanzen und der Substanzbegriffe dar, die folgenden acht Kapitel verteidigen eine stark externalistische (anti-fregeanische) Auffassung empirischer Begriffe im Allgemeinen. In beiden Werken bleiben Ontologie und Semantik stets eng verwoben.

Die vier Beiträger sprengen diese Verengungen und wenden sich unterschiedlichen Aspekten von Millikans Philosophie zu. Wolfgang Detel - einer der ersten deutschsprachigen Philosophen, der die Teleosemantik dem philosophischen Publikum auf differenzierte Weise vorgestellt hat ${ }^{12}$ - unternimmt in seinem Beitrag eine detaillierte Rekonstruktion und Evaluation von Millikans unübersichtlich anmutender Theorie natürlicher Zeichen und ihrer Verbindung zu ihrer Auffassung intentionaler Zeichen. Er gelangt zu dem Ergebnis, dass Millikans konsumentenorientierte, teleosemantische Auffassung - im Vergleich zu semiotischen, rein informationstheoretischen oder produzentenorientierten Ansätzen - einen beachtlichen Fortschritt in der Frage nach dem Zusammenhang zwischen natürlichen Zeichen und vorbegrifflichen intentionalen Repräsentationen darstellt.

Matthias Vogel, dessen in Vorbereitung befindliches Buch sich auf Millikans Teleosemantik stützt ${ }^{13}$, wendet sich in seinem Beitrag der tragenden Säule von Millikans Teleooder Biosemantik zu, nämlich ihrer Theorie der Eigenfunktionen (proper functions). Er verteidigt Millikans strikt ätiologische Auffassung von Funktionen gegen drei Vorwürfe. Diese machen geltend, dass diese Theorie ungeeignet sei, jene Funktionszuschreibungen zu rekonstruieren, die in der Biologie vorgenommen werden, dass sie explanatorisch leer sei und dass sie zur Folge habe, dass zahlreichen biologischen Phänomenen gerade nicht jene Funktionen zugeschrieben werden können, die ihnen intuitiv zugeschrieben werden.

Alex Burri, der die Übersetzung und Herausgabe einer Auswahl von sechs sprachphilosophischen Aufsätzen Millikans vorbereitet ${ }^{14}$, evaluiert in seinem Beitrag Millikans extremen semantischen Externalismus im Hinblick auf begriffliche und sprachliche Bedeutung und gelangt zu einem durchaus kritischen Ergebnis. Von den bei Millikan unterschiedenen drei Ebenen der Bedeutung kritisiert Burri die Ebene der „Konzeptionen“ (conceptions) und

12 Vgl. W. Detel, Teleosemantik. Ein neuer Blick auf den Geist?, in: Deutsche Zeitschrift für Philosophie, 49 (2001), 465-491; ders., Haben Frösche und Sumpfmenschen Gedanken? Einige Probleme der Teleosemantik, in: Deutsche Zeitschrift für Philosophie, 49 (2001), 601-626.

13 Vgl. M. Vogel, Geist und Psyche. Auf dem Weg zu einer integrativen Theorie des Mentalen, Frankfurt/M. (in Vorbereitung).

14 Vgl. R. Millikan, Biosemantik. Sprachphilosophische Aufsätze, hg. u. übers. v. A. Burri, Frankfurt/M. (erscheint 2011). 
vertritt die Ansicht, dass sich Millikans Atomismus nicht mit den biologischen Fundamenten ihres Ansatzes verträgt. Burri skizziert abschließend ein alternatives Bild von Bedeutung.

Crawford Elder - Kollege Millikans an der University of Connecticut - formuliert und verteidigt eine durch und durch realistische Metaphysik für natürliche Objekte, eine Ontologie des Common Sense, die unübersehbar von Millikans Language, Thought, and Other Biological Categories beeinflusst ist. Allerdings stimmen beide in den Ergebnissen ihrer Überlegungen nicht überein. ${ }^{15}$ Mit seinem Beitrag versucht Elder zu zeigen, dass Millikans Ontologie zwar durch und durch realistisch sein könnte und sollte, davor aber zurückschreckt, weil sie lediglich Realistin im Hinblick auf natürliche Arten, nicht aber Realistin in Bezug auf die Existenzverläufe von natürlichen Einzelobjekten ist. In ihrer Replik auf Elder weist Millikan Elders Rekonstruktion ihrer Position und insbesondere die diagnostizierte Nähe zur so genannten „Stadientheorie“ zurück. Indem sie ihren Begriff einer „Substanz“ erläutert, versucht sie zu zeigen, dass es, anders als Elder meint, einem Realismus nicht abträglich ist, wenn ein Einzelobjekt (etwa ihre Katze Tabby) Mitglied unterschiedlicher natürlicher Arten sein kann.

Elders Essay und Millikans Replik werden im Original in dem Band Millikan and Her Critics innerhalb der bekannten Blackwell-Reihe erscheinen. ${ }^{16}$ Die Arbeit der übrigen Autoren dieses Schwerpunkts sowie einige im Entstehen begriffene Forschungsprojekte zeigen, dass die Philosophie von Ruth Millikan, und zwar in ihrer ganzen Breite, zunehmend auch im deutschsprachigen Raum ein sowohl konstruktives als auch kritisches Echo auslöst. Und das ist gut so.

Markus Wild, Berlin

15 Vgl. C. Elder, Real Natures and Familiar Objects, Cambridge/Mass. 2004, xii.

16 D. Ryder u. a. (Hg.), Millikan and Her Critics, Oxford (erscheint 2012). Dieser Band wird voraussichtlich auch Beiträge von Daniel Dennett, Karen Neander, Nicholas Shea, Mohan Matthen, Peter Godfrey-Smith, John Perry, Louise Antony, Richard Fumerton, Kim Sterelny, Michael Rescorla, Robyn Carston, Robert Brandom, Jesse Prinz, Graham und Cynthia Macdonald sowie David Braddon-Mitchell enthalten. 